\title{
Discovery and validation of mucosal TNF expression combined with histological score - a biomarker for personalized treatment in ulcerative colitis
}

Jon R. Florholmen ${ }^{1,2,3}$, Kay-Martin Johnsen ${ }^{1,2^{*}}$ (D), Renate Meyer ${ }^{1}$, Trine Olsen ${ }^{1,2}, \varnothing y s t e i n$ K. Moe ${ }^{1,4}$, Petter Tandberg ${ }^{3}$, Mona D. Gundersen ${ }^{1,2}$, Jan-Magnus Kvamme ${ }^{1,2}$, Knut Johnsen ${ }^{1,4}$, Terje Løitegård ${ }^{5}$, Gabriele Raschpichler 6 , Cecilia Vold ${ }^{7}$, Sveinung W. Sørbye ${ }^{8}$ and Rasmus Goll ${ }^{1,2}$

\begin{abstract}
Background: There are no accurate markers that can predict clinical outcome in ulcerative colitis at time of diagnosis. The aim of this study was to explore a comprehensive data set to identify and validate predictors of clinical outcome in the first year following diagnosis.

Methods: Treatment naive-patients with ulcerative colitis were included at time of initial diagnosis from 2004 to 2014, followed by a validation study from 2014 to 2018. Patients were treated according to clinical guidelines following a standard step-up regime. Patients were categorized according to the treatment level necessary to achieve clinical remission: mild, moderate and severe. The biopsies were assessed by Robarts histopathology index (RHI) and TNF gene transcripts.
\end{abstract}

Results: We included 66 patients in the calibration cohort and 89 patients in the validation. Mucosal TNF transcripts showed high test reliability for predicting severe outcome in UC. When combined with histological activity (RHI) scores the test improved its diagnostic reliability. Based on the cut-off values of mucosal TNF and RHI scores from the calibration cohort, the combined test had still high reliability in the validation cohort (specificity 0.99 , sensitivity 0.44, PPV 0.89, NPV 0.87) and a diagnostic odds-ratio (DOR) of 54.

Conclusions: The combined test using TNF transcript and histological score at debut of UC can predict severe outcome and the need for anti-TNF therapy with a high level of precision. These validated data may be of great clinical utility and contribute to a personalized medical approach with the possibility of top-down treatment for selected patients.

Keywords: antiTNF, Calprotectin, Cytokines, Diagnostic odds ratio, Robarts histopathology index

\footnotetext{
* Correspondence: kay-martin.johnsen@unn.no

${ }^{1}$ Research Group of Gastroenterology and Nutrition, Department of Clinical Medicine, University of Troms $\varnothing$, Tromsø, Norway

${ }^{2}$ Department of Gastroenterology, Division of Internal Medicine, University Hospital of North Norway, Tromsø, Norway

Full list of author information is available at the end of the article
}

(c) The Author(s). 2020 Open Access This article is licensed under a Creative Commons Attribution 4.0 International License, which permits use, sharing, adaptation, distribution and reproduction in any medium or format, as long as you give appropriate credit to the original author(s) and the source, provide a link to the Creative Commons licence, and indicate if changes were made. The images or other third party material in this article are included in the article's Creative Commons licence, unless indicated otherwise in a credit line to the material. If material is not included in the article's Creative Commons licence and your intended use is not permitted by statutory regulation or exceeds the permitted use, you will need to obtain permission directly from the copyright holder. To view a copy of this licence, visit http://creativecommons.org/licenses/by/4.0/. The Creative Commons Public Domain Dedication waiver (http://creativecommons.org/publicdomain/zero/1.0/) applies to the data made available in this article, unless otherwise stated in a credit line to the data. 


\section{Background}

Ulcerative colitis (UC) is one of the two main disease entities of inflammatory bowel disease (IBD). UC is a chronic inflammatory disease believed to result from a dysregulated immune response caused by a combination of environmental and genetic factors causing loss of immunotolerance in the gut [1]. Many patients experience severe outcomes of disease with significant reduction in quality of life. The need for surgery is reported in 8 and $9.7 \%$ after 5 and 11 years, respectively [2, 3].

Definitions of clinical outcomes and prognosis in IBD are poorly defined, with little agreement on primary and secondary endpoints [4]. The IBSEN study is one of the most well-known prospective studies on clinical outcomes in UC, where the patients were divided into 4 predefined patterns of disease [2]. In a recently published review, the extent of disease and high disease activity were predictors of a more severe progression of disease [5].

The Montréal guidelines classify UC disease activity into four categories; clinical remission, mild, moderate and severe disease $[6,7]$. Different guidelines for medical and surgical treatment are available for both UC and CD in Europe and America, European Crohn's and Colitis Organization (ECCO) guidelines and American Gastroenterological Association (AGA) clinical care pathway repectively $[8,9]$. Danese et al. have created a modified algorithm with a medical step-up approach for the treatment of UC with the goal of achieving clinical remission [10]. In short, 5-ASA and local steroids are used in mild disease, with additional oral steroids, immunosuppressive and biological therapy in moderate to severe disease, consecutively. In contrast, a so-called top-down therapy has previously been documented to induce long term clinical remission of Crohn's disease [11].

From a clinical point of view, there is a need to find good predictive markers at onset of disease that enables clinicians to individually tailor therapy. There is an increasing interest for a biomarker approach. In various diseases, such as breast cancer, four gene subtypes of human epidermal growth factor receptor 2 (HER2) forms the basis of a molecular reclassification of disease according to risk factors [12]. Although there are an increasing number of reports and reviews for clinical and biochemical biomarkers at onset of disease, none have been able to predict future clinical outcome with great certainty [13-18]. In our research group we have published reports on mucosal transcript levels of tumor necrosis factor (TNF) as a biomarker for response to and when to stop anti-TNF thereapy [19-21], However, most of the studies are of retrospective design and there is a lack of validated studies of prognostic biomarkers to predict the clinical outcome in IBD with high reliability. Moreover, a personalized therapy approach initiated at the time of disease diagnosis, may have an impact on the natural course of IBD. This is so far unsettled due to the lack of long- term studies [22-24].

There is increasing knowledge of the pathophysiological events mediating the mucosal inflammation in IBD including cytokine and chemokine responses [25, 26]. So far there are few reports on how these crucial mediators can be used as biomarkers [19-22]. Therefore, the aims of this study were, first, based on a calibration cohort of newly diagnosed patients with ulcerative colitis from 2004 to 2014, to discover potential clinical, biochemical, histological and mucosal gene transcripts to predict 1 year level of treatment to obtain remission. Second, to validate these parameters in a cohort study from 2014 to 2018.

\section{Methods}

The main goal of the study was to detect and validate potential predictors of treatment level 1 year after disease onset of UC. In principle, to do a proper validation of a predictor(s) it is general accepted that this should be a two-step procedure. First, we have to study a calibration (discover) cohort, followed by a study of a validation cohort to validate the candidate predictors from the discovery study. Inclusion criteria for both the discovery and validation cohort were patients with newly diagnosed, treatment- naive UC aged $\geq 18$ years. Patients were excluded if they were lost to follow in the first year after diagnosis, patients with severe medical disease other than UC, pregnancy and lactation; and patients who first were diagnosed UC but later developed an indeterminate form of IBD.

In addition to the UC patients with newly diagnosed, treatment-naïve disease, a group of healthy subjects performing a cancer screening examination with no clinical, endoscopic or histological signs of intestinal disease were included as controls.

\section{Cohorts examined \\ Calibration cohort}

Patients attending the Gastrointestinal Unit at the University Hospital of North Norway, Tromsø, Norway, were recruited from the project Immunopathogenesis in inflammatory bowel disease in the time period January 2004 -March 2014. Validation cohort: Patients were recruited in the time period March 2014 -March 2018 attending 6 clinical centers in Norway (Gastrointestinal units at the hospitals of Kirkenes, Hammerfest, University Hospital North Norway, Tromsø, Bodø, Vestre Viken (Ringerike and Drammen)) as a part of an ongoing prospective study - Advanced Study of Inflammatory Bowel disease (ASIB- study). 


\section{Diagnosis, clinical grading and clinical outcome after 1 year}

The clinical grading of UC was based on evaluation of clinical activity at 1 year. The biopsies were histologically assessed by an experienced pathologist (SWS) using Robarts histopathology index (RHI) score [27].

The clinical outcomes of $\mathrm{UC}$ are based on the required treatment level to obtain disease remission, using the step-up algorithm guidelines ECCO and the three levels proposed by Danese et al. [10, 28] In this study we used three disease outcome levels after 1 year; mild, moderate and severe. These outcomes were defined by the treatment level needed for clinical remission; 5-ASA per oral or local (mild), need of oral steroids and/or thiopurines (moderate) and need of anti-TNF and/or surgery (severe) (see Fig. 1). Clinical remission was defined by ulcerative colitis clinical score (UCCS) < 2 [29] and/or calprotectin level $<100 \mathrm{mg} / \mathrm{kg}$ according to Feagan et al. Faecal calprotectin was measured by an ELISA kit from Calpro Norway (Oslo, Norway).

\section{Tissue samples}

Colonic mucosal biopsies were sampled from the region with the most severe inflammation. In healthy controls, biopsies were sampled from the sigmoid. Biopsy specimens for RNA extraction were immediately immersed in RNA later (Qiagen) and stored at room temperature overnight, then at $-20{ }^{\circ} \mathrm{C}$ until RNA isolation.

\section{Cytokine transcript measurements}

Total RNA was isolated from patient biopsies using Trizol until July 1, 2008; later the Allprep DNA/RNA Mini Kit (Qiagen, Hilden, Germany, Cat No: 80204) and the automated QIAcube instrument (Qiagen, Hilden, Germany) according to the manufacturer's recommendations. Quantity and purity of the extracted RNA were determined using the Qubit 3 Fluorometer (Cat No: Q33216; Invitrogen by Thermo Fisher Scientific, Waltham, MA, USA). Reverse transcription of the total RNA was performed using the QuantiTect Reverse Transcription Kit (Cat. No: 205314; Qiagen, Hilden, Germany). Mucosal TNF gene transcript was measured by real-time PCR procedures previously described in detail [30-33].

\section{Statistics}

The following factors were evaluated as predictors: extent of disease, UCDAI score and endoscopic sub-score, histological activity score, fecal calprotectin and mucosal cytokine transcripts. All baseline predictors were standardized and centered for exploring combinations of two variables. To evaluate predictors of outcome, ROC curves were constructed. Optimal cut-off values were picked by maximal Youden's J [34]. Test characteristics were derived by confusion matrices and diagnostic odds ratios [35]. A sequential test for mucosal TNF transcript and RHI score was constructed: Observations with a positive TNF test were run in a new ROC analysis for RHI score, which resulted in a two-step combined model with one cut-off value for mucosal TNF transcript and another cut-off value for RHI score following a positive TNF test.

As a global test, Kruskal Wallis one-way ANOVA was performed, then Mann-Whitney U test with Bonferroni correction. For categorical values Chi-square test with Bonferroni correction was utilized.

All statistical analyses were carried out in IBM SPSS Statistics 24 (IBM Corporation, Armonk, New York, USA).

\section{Results}

Healthy controls

Thirty-eight healthy controls were included, 13 females and 25 men aged 43-69 years. The median TNF value was 4450 copies/ $\mu g$ mRNA.

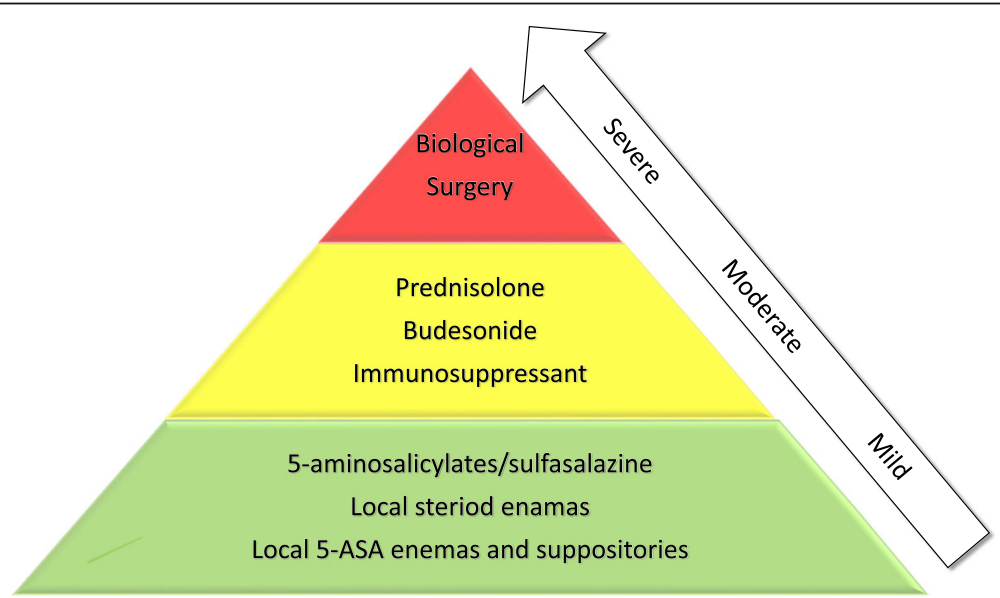

Fig. $1 \mathrm{In}$ this and the following figures data from patients with ulcerative colitis at debut of disease are grouped after 1-year treatment level outcome, Step-up algorithm according to clinical treatment outcomes (mild, moderate, severe). Modified after Danese et al., see ref. [8] 


\section{Calibration cohort}

\section{Baseline characteristics and outcome groups}

Sixty-six patients were included as a follow up mainly from an earlier report [19]. At 1 year follow-up patients were categorized into mild $(n=23)$, moderate $(n=18)$ and severe $(n=25)$ disease outcomes based on a step-up treatment level algorithm. In the moderate outcome group, no patients needed continuous steroid treatment and two patients were treated with azathioprine. In the severe outcome group, all patients were on anti-TNF treatment including one patient that later was in the need of colectomy. Sixteen patients were on concomitant treatment with azathioprine and one patient on methotrexate. An overview of baseline characteristics for each outcome group is shown in Table 1. There were significant differences between the three treatment groups for mucosal TNF and UCDAI scores $(p<0.017)$.

\section{Discovery of potential biomarkers}

With three defined treatment outcomes we made two sets of ROC curves, one set to discriminate between mild and moderate/severe and one set to discriminate between mild/moderate and severe. There were no baseline predictors that showed good test performance for discriminations between mild and moderate/severe (data not shown). However, there was a tendency towards increasing concentrations of the mucosal TNF transcripts with increasing treatment level (Fig. 2a).

\section{Severe outcome}

Baseline predictors of severe outcome are shown in Table 1 and Fig. 3 presenting clinical parameters (Calprotectin, UCDAI, Mayo endoscopic score), RHI score and mucosal TNF transcripts. Selected predictors including cut off values are shown in Table 3. Of individual factors, mucosal TNF transcript had the best test performance with a sensitivity, specificity and diagnostic odds ratio (DOR) of $0.81,0.91$ and 43 respectively. Clinical data including fecal calprotectin, UCDAI and RHI -score, yielded a high sensitivity but poor specificity (Table 2, Fig. 3), and therefore a poorer test performance than mucosal TNF transcript. To increase the test performance, we then combined mucosal TNF transcript and RHI score in a sequential setup: subjects with mucosal TNF transcript above cut-off were subjected to a second ROC curve using RHI score as predictor. The combined sequential test of mucosal TNF transcript and RHI score showed a superior test performance for specificity and DOR, however lower sensitivity (Table 2). No other clinical, biochemical, histological or immunological combinations could improve the test performance of prediction of severe outcome (supplement material Fig. 4).

\section{Validation cohort \\ Baseline characteristics and outcome groups}

At one year follow up patients were categorized into mild $(n=36)$, moderate $(n=31)$ and severe $(n=22)$ disease outcomes based on a step-up treatment level

Table 1 Baseline characteristics of patients in the calibration cohort with ulcerative colitis according to one-year treatment outcome level

\begin{tabular}{llll}
\hline Patients groups & Mild $\mathbf{N = 2 3}$ & Moderate $\mathbf{N = 1 8}$ & Severe $\boldsymbol{N}=\mathbf{2 5}$ \\
\hline $\begin{array}{l}\text { Age med (IQR) } \\
\text { Sex }\end{array}$ & $41(35-54)$ & $35(24-55)$ & $41(27-54)$ \\
$\quad$ Female & $15(65 \%)$ & $7(39 \%)$ & $9(36 \%)$ \\
$\quad$ Male & $8(35 \%)$ & $11(61 \%)$ & $16(64 \%)$ \\
Colonic area involved & & & $3(12 \%)$ \\
$\quad$ Proctitis & $9(39 \%)$ & $3(17 \%)$ & $10(40 \%)$ \\
Left side & $9(39 \%)$ & $7(39 \%)$ & $12(48 \%)$ \\
$\quad$ Extensive & $5(22 \%)$ & $8(44 \%)$ & 12 \\
Smoking & 14 & 21 & $2(10 \%)$ \\
Current smoker & $4(29 \%)$ & $2(17 \%)$ & $18(90 \%)$ \\
Non-smoker & $10(71 \%)$ & $10(83 \%)$ & $26,900(18700-40,400)$ \\
Mucosal TNF* & $10,500(4600-11,900)$ & $12,000(8000-17,200)$ & $12(9-12)$ \\
UCDAl med (IQR)* at debut & $7(5-8)$ & $9(8-12)$ & $2300(670-2500)$ \\
Calprotectin med (IQR) & $590(400-1100)$ & $790(470-1540)$ & $9(7-12)$ \\
RHI med (IQR) & $9(5-10)$ & $7(6-10)$ & $0(0-2)$ \\
UCCS score 1-year med (IQR) & $0(0-0)$ & $0(0-2)$ & $25(0-160)$ \\
Calprotectin 1-year med (IQR) & $60(25-85)$ & $50(25-100)$ &
\end{tabular}

${ }^{*} p<0,017$ between groups, Mann-Whitney $\mathrm{U}$ test with Bonferroni correction

Med (IQR) Median (Interquartile range), RHI Robarts histopathology index. Mucosal TNF in copies/Mg RNA: Fecal calprotectin in mg/kg 

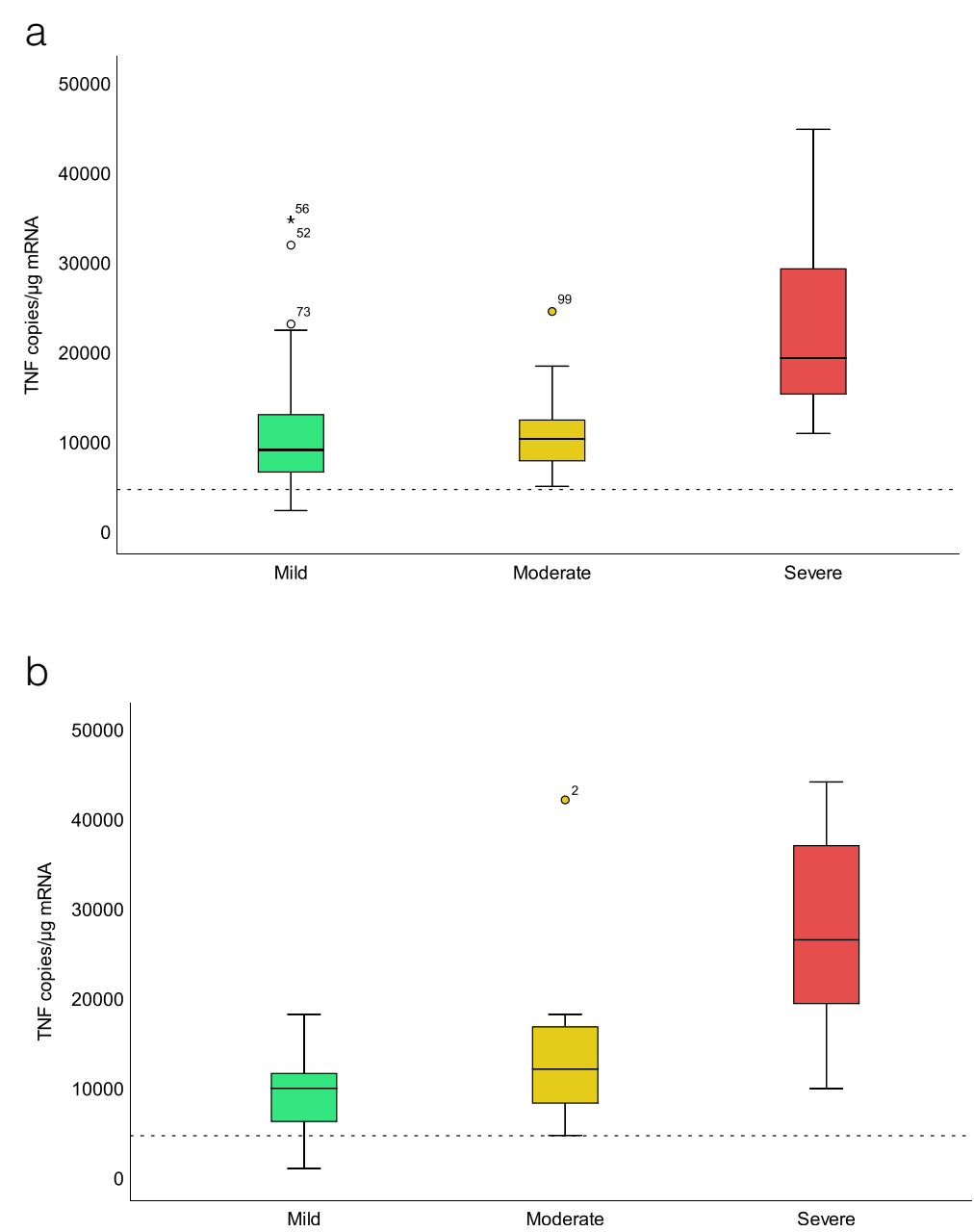

Fig. 2 Mucosal TNF transcript in treatment outcome groups and in healthy normal controls in the calibration cohort (a) and the validation group (b)

algorithm. In the moderate outcome group, no patients needed continuous steroid treatment and five subjects were treated with azathioprine. In the severe outcome group, 22 patients were on anti-TNF treatment whereas two of these patients were later in the need of colectomy. Thirty-eight healthy controls were included. An overview of baseline characteristics for each outcome group is shown in Table 3. There were significant differences between the three treatment groups for mucosal TNF, UCDAI, RHI scores and fecal calprotectin (Table 3, Fig. 2b).

\section{Validation of predictors of severe outcome}

The cut off values from the discovery study (TNF $\geq 18,000$, RHI $\geq 9$ ) were used for test performance. The baseline predictors of severe outcome presenting mucosal TNF transcripts and RHI score are shown in Table 4. Mucosal TNF transcript had a test performance with sensitivity, specificity and DOR of 0.5, 0.9 and 9 respectively. RHI transcript had a test performance with sensitivity, specificity and DOR of
0.72, 0.69 and 6, respectively. When combined TNF and RHI the specificity increased to high 0.99 , whereas the DOR was still high as 54 . Moreover, the low sensitivity of 0.44 represents most likely the overlapping TNF and RHI score values to the mild/moderate outcome groups (Table 3).

\section{Discussion}

We present a combined discovery study (from 2004) and a validation study (from 2014) in a prospective design (the transomic Advanced Study of Inflammatory Bowel Disease) where clinical, biochemical, histological and transcript data where retrospectively tested to identify biomarkers of clinical outcome 1 year after disease diagnosis of UC. Mucosal TNF transcripts showed high test reliability for predicting severe outcome after 1 year in $\mathrm{UC}$ in both studies but was not ideal to discriminate between mild, moderate and severe disease. Moreover, when the TNF transcripts were combined with histological activity (RHI) scores, the test improved its 


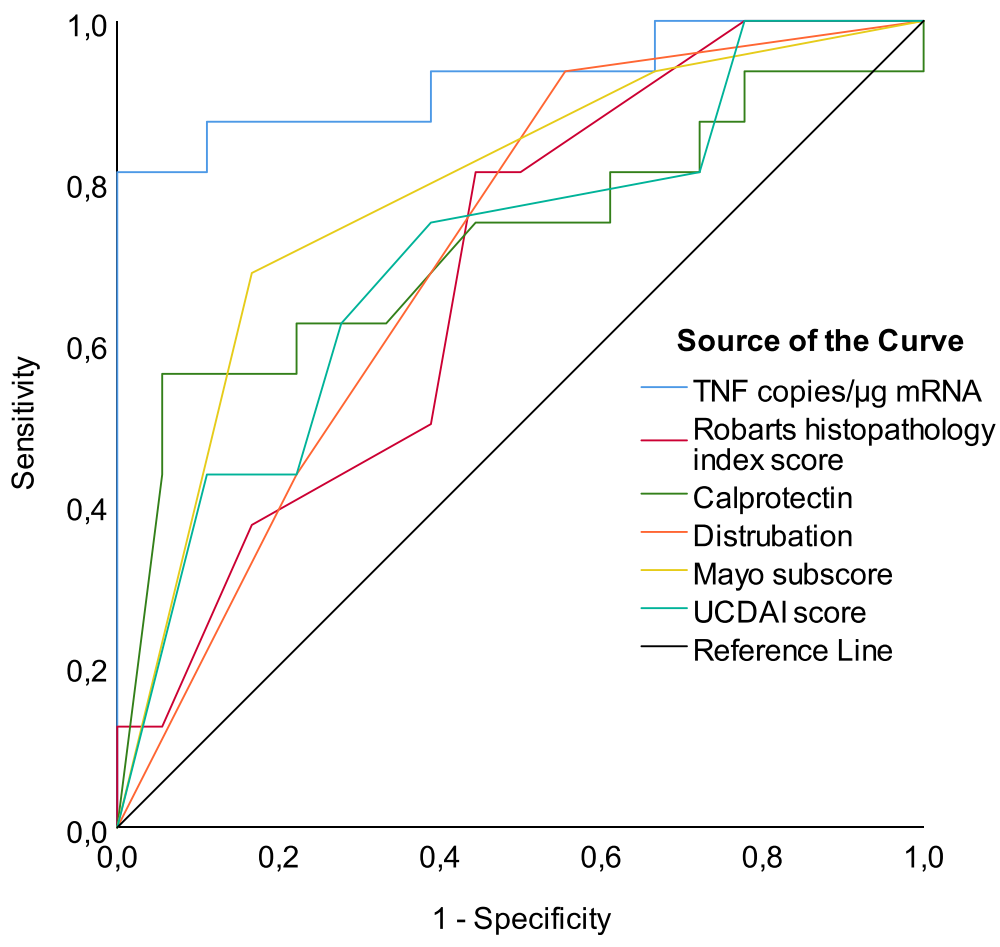

Fig. 3 ROC curves of predictors of severe outcome in calibration cohorte

diagnostic reliability. Mucosal cut-off values for TNF and RHI scores determined in the calibration cohort displayed a high test performance with specificity of 0.99 and a diagnostic odds-ratio (DOR) of 54 in the prospective validation study. Thus, mucosal TNF transcript combined with a histological score at debut of disease can likely identify patients who experience severe outcomes during the first year. This is an important step towards personalizing treatment in IBD and may be used as a criterion for selecting candidates for top-down treatment of anti-TNF. However, this awaits further studies.

We have tested a broad spectrum of potential factors that could, alone, or in combinations, predict clinical outcome in the first year of diagnosis. The clinical outcomes were defined as the highest treatment level required for achieving disease remission during the first year of disease, in a step-up treatment approach. The broad/wide selection of variables including various combinations did not have the necessary precision to discriminate between mild, moderate and severe outcomes. However mucosal TNF transcript in combination with the histological RHI score was able to predict, with high precision, the most severe colitis outcomes needing biological or surgical treatment, within the first year of disease. The validated cut-off values (TNF $\geq 18,000, \mathrm{RHI} \geq$ 9) showed a high specificity to predict severe outcome and a DOR as high as 54. From a clinical point of view, these cut-off values indicate a need of anti-TNF therapy during the first year after diagnosis with high reliability, and therefore of high clinical value and utility in the management of IBD/UC. In order to use a biomarker for selection for top-down treatment, a high PPV is

Table 2 Factors at debut of ulcerative colitis in the calibration cohort to predict severe treatment outcomes at one year of disease

\begin{tabular}{|c|c|c|c|c|c|c|c|}
\hline Factors & Youden's J & Cut-off value & Sensitivity & Specificity & PPV & NPV & DOR \\
\hline $\mathrm{TNF}^{\mathrm{a}}$ & 0,72 & $\geq 18,000$ & 0,81 & 0,91 & 0,85 & 0,89 & 43 \\
\hline $\mathrm{RH} \mathrm{I}^{\mathrm{a}}$ & 0,23 & $\geq 9$ & 0,71 & 0,52 & 0,48 & 0,74 & 3 \\
\hline Combined TNF RHI & 0,57 & $\geq 18,000$ and $\geq 9$ & 0,57 & 1 & 1 & 0,79 & $\infty$ \\
\hline UCDAI & 0,4 & $\geq 9$ & 0,79 & 0,61 & 0,54 & 0,83 & 6 \\
\hline Mayo subscore & 0,45 & 3 & 0,72 & 0,73 & 0,62 & 0,81 & 7 \\
\hline Calprotectin & 0,51 & $\geq 2000$ & 0,6 & 0,91 & 0,86 & 0,72 & 15 \\
\hline
\end{tabular}

Diagnostic odds ratio PPV: Positive predictive value NPV Negative predictive value

${ }^{a}$ copies/ug mRNA ${ }^{b}$ Robarts histopathology index score 
Table 3 Baseline characteristics of patients with ulcerative colitis in the validation cohort according to one-year treatment outcome level

\begin{tabular}{|c|c|c|c|}
\hline Patient groups & Mild $N=36$ & Moderate $N=31$ & Severe $N=22$ \\
\hline Age med (IQR) & $36(24-49)$ & $30(24-41)$ & $26(22-47)$ \\
\hline \multicolumn{4}{|l|}{ Sex } \\
\hline Female & $17(47 \%)$ & $8(26 \%)$ & $10(46 \%)$ \\
\hline Male & 19 (53\%) & $23(74 \%)$ & $12(54 \%)$ \\
\hline \multicolumn{4}{|l|}{ Colonic area involved } \\
\hline Proctitis & $5(14 \%)$ & $1(3 \%)$ & $1(4 \%)$ \\
\hline Left side & $25(69 \%)$ & $18(58 \%)$ & $10(46 \%)$ \\
\hline Extensive & $6(17 \%)$ & $12(39 \%)$ & $11(50 \%)$ \\
\hline Smoking & 28 & 21 & 12 \\
\hline Current smoker & $1(4 \%)$ & $2(10 \%)$ & $1(8 \%)$ \\
\hline Non-smoker & $27(96 \%)$ & 19 (90\%) & $11(92 \%)$ \\
\hline Mucosal TNF* & $8800(6100-12,800)$ & $10,500(7400-13,200)$ & $17,400(15100-26,800)$ \\
\hline UCDAI med (IQR)* at debut & $7(5-9)$ & $9(8-11)$ & $10(7-11)$ \\
\hline Calprotectin med $(\mathrm{QQR})^{*}$ & $570(200-970)$ & $1000(340-2000)$ & $1100(830-1400)$ \\
\hline $\mathrm{RHI}$ med $(\mathrm{IQR})^{*}$ & $6(2-10)$ & $6(4-11)$ & $14(9-27)$ \\
\hline UCCS score 1-year med (IQR) & $0(0-0)$ & $0(0-0)$ & $0(0-8)$ \\
\hline Calprotectin 1-year med (IQR) & $40(25-94)$ & $50(0-140)$ & $25(20-60)$ \\
\hline
\end{tabular}

${ }^{*} p<0,017$ between groups, Mann-Whitney $\mathrm{U}$ test with Bonferroni correction

Med (IQR): median (Interquartile range); RHI: Robarts histopathology index. Mucosal TNF in copies/ $\mu$ g RNA; Fecal calprotectin in mg/kg

necessary to avoid excessive use of biologics. Our proposed biomarker shows a PPV of 0.89 meaning that 9 out of 10 positives will be correctly identified as severe outcome.

A step-up treatment approach represents wellestablished international guidelines [8-10]. One drawback of this approach is that patients in the severe outcome group often experience a period of poor response during the gradual escalation of treatment intensity until an adequate response is obtained. In some cases, one may lose an important window of opportunity for optimal effect of biologics leading to permanent structural damage and/or need of surgery. The impact of early treatment before development of severe disease is not completely investigated. However, the top down approach published by D'Haens et al. indicated that immunosuppressive therapy was superior to a step-up approach in patients with Crohn's disease [36]. Moreover, it is well documented that induction of treatment to remission reduces later hospitalization, whereas conflicting results exist for colectomy in two studies [37, 38].

The use of molecular data from the mucosa represents a novel approach and is an easily available tool, with high utility for clinicians to individually tailor therapy in UC. Endoscopic biopsies are routinely taken at diagnosis and surveillance of IBD. Thus, the logistics of measuring mucosal TNF transcript are simple, as biopsies are readily available and samples do not require freezing prior to analysis [31].

Our study contributes with new knowledge in the scientific field of personalized therapy in UC $[15,16]$. We know that treatment to remission improves long-term clinical outcome $[39,40]$. The main question is: Can a top-down therapy of the most severe forms of disease have an effect on the natural course of disease? This awaits future studies.

The strength of this prospective designed, combined discovery and validation study is that we have

Table 4 Factors at debut of ulcerative colitis in the validation cohort to predict severe treatment outcomes at one year of disease based on cut off values from the discovery cohort

\begin{tabular}{llllllll}
\hline Factors & Youden's J & Cutt off value & Sensitivity & Specificity & PPV & NPV & DOR \\
\hline $\mathrm{TNF}^{\mathrm{a}}$ & 0,40 & $\geq 18,000$ & 0,50 & 0,90 & 0,56 & 0,87 & 9 \\
$\mathrm{RH} \mathrm{b}^{\mathrm{b}}$ & 0,41 & $\geq 9$ & 0,72 & 0,69 & 0,38 & 0,90 & 6 \\
Combined TNF RHI & 0,43 & $18,000 \geq 9$ & 0,44 & 0,99 & 0,89 & 0,87 & 54 \\
\hline
\end{tabular}

DOR Diagnostic odds ratio, PPV Positive predictive value NPV Negative predictive value

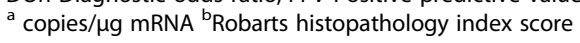


retrospectively searched for and validated biomarkers for treatment at debut of UC, using a broad search of clinical, histological and analytical factors including mucosal immune transcripts. Moreover, this is part of the transomic Advanced Study of Inflammatory Bowel disease (ASIB) study where parallel studies of the epigenome, transcriptome, proteome and metabolome are ongoing [33, 41-45]. .This transomic approach at debut of UC will be performed and correlated to long-term clinical outcome. Therefore, the upcoming transomic data from the ASIB study and from several ongoing studies such as the PREDICTS study will not only search for therapeutic but also prognostic and natural course biomarkers [17]. The weakness of the study includes the lack of endoscopic diagnosis at 1 year, which would have given insight into endoscopic status and endoscopic remission rates according to treatment levels. Additionally, the decision to use or not use steroids at time of diagnosis is dependent on the subjective decision of the clinicians. This may be one explanation for the small differences detected between the mild and moderate treatment group.

\section{Conclusion}

The combined information of mucosal TNF transcription and histological score at debut of UC can predict severe outcome and the need for anti-TNF therapy. This is of great clinical utility and may contribute to a personalized medicine approach in UC.

\section{Supplementary information}

Supplementary information accompanies this paper at https://doi.org/10. 1186/s12876-020-01447-0.

Additional file 1: Figure 4. Supplement figure with ROC curves of predictors of mild outcome from calibration cohort

\section{Abbreviations}

RHI: Robarts histopathology index; DOR: Diagnostic odds-ratio; UC: Ulcerative colitis; IBD: Inflammatory bowel disease; TNF: Tumor necrosis factor; ASIBstudy: Advanced Study of Inflammatory Bowel disease; UCCS: Ulcerative colitis clinical score

\section{Acknowledgments}

The publication charges for this article have been funded by a grant from the publication fund of UiT The Arctic University of Norway. We thank Ingrid Christiansen, Marian Remijn and Line Wilsgaard for expert technical assistance.

\section{Authors' contributions}

Planning and conducting: JRF, KMJ, RG, KJ, RM, TO, SWS, ØKM, PT, MDG, JMK, TL, GR, CV Collecting or interpreting data: JRF, KMJ, RG, KJ, RM, TO, SWS, $\varnothing K M, P T, M D G, J M K, T L, G R, C V$. Drafting of manuscript: JRF, KMJ, RG, KJ, RM, TO, SWS, ØKM, PT, MDG, JMK, TL, GR, CV. The authors read and approved the final manuscript.

\section{Funding}

This work was supported by Northern Norway Regional Health Authority, ID SFP-50-04, SFP-888-09 and SFP-1136-13.
Availability of data and materials

Data are available from the authors upon reasonable request due to privacy/ ethical restrictions.

Ethics approval and consent to participate

All participants were informed and signed a written consent to participate and publication.

Approval including the use of biobank was granted by the Regional Committee of Medical Ethics of Northern Norway Ref no: 14/2004 and 1349/ 2012.

\section{Consent for publication}

All authors have approved the final manuscript for publication.

\section{Competing interests}

None declared from all authors.

\section{Author details}

${ }^{1}$ Research Group of Gastroenterology and Nutrition, Department of Clinical Medicine, University of Tromsø, Tromsø, Norway. ${ }^{2}$ Department of Gastroenterology, Division of Internal Medicine, University Hospital of North Norway, Tromsø, Norway. ${ }^{3}$ Department of Internal Medicine, Vestre Viken Hospital, Hønefoss, Norway. ${ }^{4}$ Department of Internal Medicine, Hammerfest Hospital, Hammerfest, Norway. ${ }^{5}$ Department of Gastroenterology, Vestre Viken Hospital, Drammen, Norway. ${ }^{6}$ Department of Internal Medicine, Kirkenes, Norway. ${ }^{7}$ Department of Gastroenterology, Nordland Hospital, Bodø, Norway. ${ }^{8}$ Department of Pathology, University Hospital of North Norway, Tromsø, Norway.

Received: 22 April 2020 Accepted: 9 September 2020

Published online: 02 October 2020

\section{References}

1. Zhang YZ, Li YY. Inflammatory bowel disease: pathogenesis. World J Gastroenterol. 2014;20:91-9.

2. Henriksen M, Jahnsen J, Lygren I, et al. Ulcerative colitis and clinical course: results of a 5-year population-based follow-up study (the IBSEN study). Inflamm Bowel Dis. 2006;12:543-50.

3. Solberg IC, Hoivik ML, Cvancarova M, et al. Risk matrix model for prediction of colectomy in a population-based study of ulcerative colitis patients (the IBSEN study). Scand J Gastroenterol. 2015;50:1456-62.

4. Williet N, Sandborn WJ, Peyrin Biroulet L. Patient-reported outcomes as primary end points in clinical trials of inflammatory bowel disease. Clin Gastroenterol Hepatol. 2014;12:1246-1256.e6.

5. da Silva BC, Lyra AC, Rocha R, et al. Epidemiology, demographic characteristics and prognostic predictors of ulcerative colitis. World J Gastroenterol. 2014;20:9458-67.

6. Silverberg MS, Satsangi J, Ahmad T, et al. Toward an integrated clinical, molecular and serological classification of inflammatory bowel disease: report of a working party of the 2005 Montreal world congress of gastroenterology. Can J Gastroenterol. 2005;19 Suppl A:5A-36A.

7. Satsangi J, Silverberg MS, Vermeire $S$, et al. The Montreal classification of inflammatory bowel disease: controversies, consensus, and implications. Gut. 2006;55:749-53.

8. Dassopoulos T, Cohen RD, Scherl EJ, et al. Ulcerative colitis care pathway. Gastroenterology. 2015;149:238-45.

9. Magro F, Gionchetti P, Eliakim R, et al. Third European evidence-based consensus on diagnosis and Management of Ulcerative Colitis. Part 1: definitions, diagnosis, extra-intestinal manifestations, pregnancy, cancer surveillance, surgery, and Ileoanal pouch disorders. J Crohns Colitis. 2017;11:649-70.

10. Danese $S$, Siegel CA, Peyrin-Biroulet L. Review article: integrating budesonide-MMX into treatment algorithms for mild-to-moderate ulcerative colitis. Aliment Pharmacol Ther. 2014;39:1095-103.

11. D'Haens GR. Top-down therapy for IBD: rationale and requisite evidence. Nat Rev Gastroenterol Hepatol. 2010;7:86-92.

12. Wolff $A C$, Hammond ME, Hicks DG, et al. Recommendations for human epidermal growth factor receptor 2 testing in breast cancer: American Society of Clinical Oncology/College of American Pathologists clinical practice guideline update. J Clin Oncol. 2013;31:3997-4013. 
13. Choung RS, Princen F, Stockfisch TP, et al. Serologic microbial associated markers can predict Crohn's disease behaviour years before disease diagnosis. Aliment Pharmacol Ther. 2016;43:1300-10.

14. Hamilton AL, Kamm MA, De Cruz P, et al. Serologic antibodies in relation to outcome in postoperative Crohn's disease. J Gastroenterol Hepatol. 2017;32: 1195-203.

15. Spekhorst LM, Imhann F, Festen EAM, et al. Cohort profile: design and first results of the Dutch $\mathrm{BBD}$ biobank: a prospective, nationwide biobank of patients with inflammatory bowel disease. BMJ Open. 2017;7:e016695.

16. Stevens TW, Matheeuwsen M, Lonnkvist MH, et al. Systematic review: predictive biomarkers of therapeutic response in inflammatory bowel disease-personalised medicine in its infancy. Aliment Pharmacol Ther. 2018; 48:1213-31.

17. Porter CK, Riddle MS, Gutierrez RL, et al. Cohort profile of the PRoteomic evaluation and discovery in an IBD cohort of tri-service subjects (PREDICTS) study: rationale, organization, design, and baseline characteristics. Contemp Clin Trials Commun. 2019;14:100345.

18. Dulai PS, Peyrin-Biroulet $L$, Danese $S$, et al. Approaches to integrating biomarkers into clinical trials and care pathways as targets for the treatment of inflammatory bowel diseases. Gastroenterology. 2019;157:1032-1043.e1.

19. Olsen T, Goll R, Cui G, et al. TNF-alpha gene expression in colorectal mucosa as a predictor of remission after induction therapy with infliximab in ulcerative colitis. Cytokine. 2009;46:222-7.

20. Rismo R, Olsen T, Cui G, et al. Normalization of mucosal cytokine gene expression levels predicts long-term remission after discontinuation of antiTNF therapy in Crohn's disease. Scand J Gastroenterol. 2013;48:311-9.

21. Johnsen KM, Goll R, Hansen $V$, et al. Repeated intensified infliximab induction - results from an 11-year prospective study of ulcerative colitis using a novel treatment algorithm. Eur J Gastroenterol Hepatol. 2017;29:98-104.

22. Flamant $M$, Roblin X. Inflammatory bowel disease: towards a personalized medicine. Therap Adv Gastroenterol. 2018;11:1756283×17745029.

23. Siegel CA, Refocusing IBD. Patient management: personalized, proactive, and patient-centered care. Am J Gastroenterol. 2018;113:1440-3.

24. Weimers P, Munkholm P. The natural history of IBD: lessons learned. Curr Treat Options Gastroenterol. 2018;16:101-11.

25. de Souza HSP. Etiopathogenesis of inflammatory bowel disease: today and tomorrow. Curr Opin Gastroenterol. 2017;33:222-9.

26. Kim DH, Cheon JH. Pathogenesis of inflammatory bowel disease and recent advances in biologic therapies. Immune Netw. 2017;17:25-40.

27. Mosli MH, Feagan BG, Zou G, et al. Development and validation of a histological index for UC. Gut. 2017;66:50-8.

28. Dignass A, Lindsay JO, Sturm A, et al. Second European evidence-based consensus on the diagnosis and management of ulcerative colitis part 2 current management. J Crohns Colitis. 2012;6:991-1030.

29. Feagan BG, Greenberg GR, Wild G, et al. Treatment of ulcerative colitis with a humanized antibody to the a4 $\beta 7$ integrin. N Engl J Med. 2005;352:2499-507.

30. Olsen T, Goll R, Cui G, et al. Tissue levels of tumor necrosis factor-alpha correlates with grade of inflammation in untreated ulcerative colitis. Scand J Gastroenterol. 2007;42:1312-20.

31. Cui G, Olsen T, Christiansen I, et al. Improvement of real-time polymerase chain reaction for quantifying TNF-alpha mRNA expression in inflamed colorectal mucosa: an approach to optimize procedures for clinical use. Scand J Clin Lab Invest. 2006;66:249-59.

32. Olsen T, Rismo R, Gundersen MD, et al. Normalization of mucosal tumor necrosis factor-alpha: a new criterion for discontinuing infliximab therapy in ulcerative colitis. Cytokine. 2016;79:90-5.

33. Diab J, Al-Mahdi R, Gouveia-Figueira S, et al. A quantitative analysis of colonic mucosal Oxylipins and Endocannabinoids in treatment-naive and deep remission ulcerative colitis patients and the potential link with cytokine gene expression. Inflamm Bowel Dis. 2019;25:490-7.

34. Youden WJ. Index for rating diagnostic tests. Cancer. 1950;3:32-5.

35. Glas AS, Lijmer JG, Prins MH, et al. The diagnostic odds ratio: a single indicator of test performance. J Clin Epidemiol. 2003;56:1129-35.

36. D'Haens G, Baert F, van Assche G, et al. Early combined immunosuppression or conventional management in patients with newly diagnosed Crohn's disease: an open randomised trial. Lancet. 2008;371:660-7.

37. Colombel JF, Rutgeerts P, Reinisch W, et al. Early mucosal healing with infliximab is associated with improved long-term clinical outcomes in ulcerative colitis. Gastroenterology. 2011;141:1194-201.

38. Burisch J, Kiudelis G, Kupcinskas L; Epi-IBD group, et al. Natural disease course of Crohn's disease during the first 5 years after diagnosis in a
European population-based inception cohort: an Epi-IBD study. Gut. 2019; 68:423-33.

39. Rutgeerts $P$, Vermeire $S$, Van Assche G. Mucosal healing in inflammatory bowel disease: impossible ideal or therapeutic target? Gut. 2007;56:453-5.

40. Arias MT, Vande Casteele N, Vermeire S, et al. A panel to predict long-term outcome of infliximab therapy for patients with ulcerative colitis. Clin Gastroenterol Hepatol. 2015;13:531-8.

41. Schniers A, Anderssen E, Fenton CG, et al. The proteome of ulcerative colitis in colon biopsies from adults - optimized sample preparation and comparison with healthy controls. Proteomics Clin Appl. 2017;11. https://doi. org/10.1002/prca.201700053. https://onlinelibrary.wiley.com/action/ showCitFormats?doi=10.1002\%2Fprca.201700053.

42. Taman H, Fenton CG, Hensel IV, et al. Genome-wide DNA methylation in treatment-naive ulcerative colitis. J Crohns Colitis. 2018;12:1338-47.

43. Taman H, Fenton CG, Hensel IV, et al. Transcriptomic landscape of treatment-naive ulcerative colitis. J Crohns Colitis. 2018;12:327-36.

44. Schniers A, Goll R, Pasing Y, et al. Ulcerative colitis: functional analysis of the in-depth proteome. Clin Proteomics. 2019;16:4.

45. Diab J, Hansen T, Goll R, et al. Lipidomics in ulcerative colitis reveal alteration in mucosal lipid composition associated with the disease state. Inflamm Bowel Dis. 2019;25:1780-7.

\section{Publisher's Note}

Springer Nature remains neutral with regard to jurisdictional claims in published maps and institutional affiliations.
Ready to submit your research? Choose BMC and benefit from:

- fast, convenient online submission

- thorough peer review by experienced researchers in your field

- rapid publication on acceptance

- support for research data, including large and complex data types

- gold Open Access which fosters wider collaboration and increased citations

- maximum visibility for your research: over $100 \mathrm{M}$ website views per year

At BMC, research is always in progress.

Learn more biomedcentral.com/submissions 